\title{
miR-153 inhibits epithelial-to-mesenchymal transition in hepatocellular carcinoma by targeting Snail
}

\author{
WENFEI XIA ${ }^{1 *}$, XIAOPENG MA ${ }^{2 *}$, XINGRUI LI $^{1}$, HONG DONG $^{1}$, JILIN YI $^{1}$, \\ WEIXIA ZENG ${ }^{3}$ and ZHIFANG YANG ${ }^{1}$ \\ ${ }^{1}$ Department of General Surgery, Tongji Hospital, Tongji Medical College of Huazhong University of Science \\ and Technology, Wuhan, Hubei; ${ }^{2}$ Department of General Surgery, Anhui Provincial Hospital Affiliated \\ to Anhui Medical University, Hefei, Anhui; ${ }^{3}$ Laura Biotech Co., Ltd., Guangzhou, Guangdong, P.R. China
}

Received January 20, 2015; Accepted February 19, 2015

DOI: $10.3892 / o r .2015 .4008$

\begin{abstract}
Epithelial-to-mesenchymal transition (EMT) has been implicated as a dynamic cellular process in embryonic development and invasion of human cancers. Snaill is a critical convergence hub in EMT regulation which transcriptionally represses E-cadherin expression. Currently, published data indicate that upregulation of Snail is mainly due to transcriptional activation and regulation of protein stability and cellular location. However, whether there is an alternative regulatory mechanism remains unclear. Our study showed that the expression of miR-153 was noticeably downregulated in hepatocellular carcinoma (HCC) cell lines and tissues, compared with normal liver epithelial cells (NLCs) and matched adjacent normal HCC tissues. Ectopic expression of miR-153 inhibited the migration and invasion ability of HCC cells, while suppression of miR-153 rescued this inhibitory effect. In addition, upregulation of miR-153 in HCC cells resulted in a decrease in epithelial markers, E-cadherin and $\alpha$-catenin, and an increase in mesenchymal markers, $\mathrm{N}$-cadherin and vimentin, and vice versa. Moreover, we demonstrated that miR-153 downregulated Snail expression by directly targeting the 3'-untranslated region (3'UTR) of Snail. Taken together, our results suggest that miR-153 plays a critical role in suppressing EMT and $\mathrm{HCC}$ progression by direct suppression of Snail expression.
\end{abstract}

\section{Introduction}

Epithelial-to-mesenchymal transition (EMT) was originally identified as a dynamic cellular process in embryonic develop-

Correspondence to: Dr Zhifang Yang, Department of General Surgery, Tongji Hospital, Tongji Medical College of Huazhong University of Science and Technology, Wuhan, Hubei 430030, P.R. China

E-mail: zhifang_yang@126.com

*Contributed equally

Key words: miR-153, epithelial-to-mesenchymal transition, Snail, hepatocellular carcinoma ment, during which epithelial cells lose their epithelial cell characteristics and are converted to motile mesenchymal cells $(1,2)$. During the past decade, EMT was thought to underlie metastasis by promoting the achievement of migratory ability and to play prominent roles in invasion and metastasis of human cancers, including hepatocellular carcinoma (HCC) (2-4). The hallmarks of EMT include loss of epithelial markers such as E-cadherin and $\alpha$-catenin, and acquisition of mesenchymal markers such as $\mathrm{N}$-cadherin, fibronectin, as well as vimentin (1). Among these proteins, E-cadherin has been considered to be a critical switch in EMT. Transcription factors including members of the Snail family (Snail and Slug), bHLH factors, Twist and the ZEB family (ZEB1 and ZEB2) are main molecules involved in orchestrating EMT by targeting the promoter of E-cadherin gene and abolishing its expression (5-8).

Snail is the first identified and most important transcriptional repressor of E-cadherin. Snail-induced EMT is partly due to the direct repression of the E-cadherin promoter. Recent research indicates that the effects of Snail on epithelial cells include repressing E-cadherin, promoting the expression of other transcriptional factors such as ZEB1 (9), and activation of the transforming growth factor- $\beta$ signaling pathway (10). As a convergence point in EMT induction, Snail is highly unstable and is strictly under the regulation of an integrated and complex signaling network at the transcriptional and post-transcriptional level in different cellular contexts. This network includes epidermal growth factor (EGF), fibroblast growth factor (FGF), hepatocyte growth factor (HGF), transforming growth factor $\beta$ (TGF $\beta$ ), bone morphogenetic proteins (BMPs), phosphatidylinositol 3-kinase (PI3-K), mitogen-activated protein kinases (MAPKs), NFאB pathways, WNTs and Notch (11-13). At the post-transcriptional level, Snail is dually regulated by protein stability and cellular location, which is mainly governed by GSK $3 \beta$, PAK 1 , the zinc-finger transporter LIV1 and hypoxia-inducible factor $\alpha$ (HIF-1 $\alpha)$ (14-17). Although activation of the multiple abovementioned signaling pathways and molecules can contribute to the concentration, localization and function of Snail, it is reasonable to question why cancer cells upregulate Snail protein via multiple steps (such as activated transcription, phosphorylation, nuclear/cytoplasmic translocation, and degradation) rather than stop its 
synthesis at the translational level. Therefore, we hypothesized that alternative regulatory mechanisms of Snail may exist.

MicroRNAs (miRNAs) are a class of small non-coding RNAs with 20-22 nucleotides that are highly conserved throughout metazoan species (18). MicroRNAs suppress gene expression by inhibition of translation or facilitation of mRNA degradation by binding to the 3'-untranslated region (3'UTR) of a target mRNA with perfect or near perfect complementarity $(19,20)$. Recent research has highlighted the significance of microRNAs as powerful modulators of EMT and cancer metastasis $(21,22)$. For example, it was reported that the miR-200 family and miR-205 inhibit EMT and cell migration by directly targeting E-cadherin repressors, ZEB1 and ZEB2 (23-25). However, whether there are other microRNAs that regulate the expression of Snail remain largely unknown.

In the present study, we measured the expression level of miR-153 in HCC cell lines and tissues and compared it with that in normal tissues. We also investigated the effect of miR-153 overexpression on the EMT phenotypes of tumor cells. We found that miR-153 inhibits HCC migration and invasion by directly targeting the 3'UTR of the Snail mRNA, consequently reducing the expression of vimentin, $\mathrm{N}$-cadherin and vimentin, and increasing the expression of E-cadherin as well as $\alpha$-catenin. Our results suggest that miR-153 may play a crucial role in the EMT process and HCC progression through downregulation of Snail.

\section{Materials and methods}

Tissue specimens and cell cultures. Prior written consent was obtained from patients, and approval for the use of clinical materials for research purposes was obtained from the Tongji Hospital Institutional Ethics Board and was conducted in compliance with the Declaration of Helsinki Ethical Principles for Clinical Studies. The $14 \mathrm{HCC}$ tissues and adjacent non-cancerous tissues used in this study were obtained from patients who underwent radical operations at Tongji Hospital of Tongji Medical College of Huazhong University of Science and Technology.

Cell culture. Primary cultures of normal liver epithelial cells (NLCs) were established from fresh biopsies of the adjacent non-cancerous liver tissue; these cultures were maintained in complete keratinocyte serum-free medium (Invitrogen, Carlsbad, CA, USA). HCC cell lines, including HepG2, MHCC97H, Hep3B, MHCC97L, HCCC9810, BEL-7402, Huh7 and QGY-7703, were grown in Dulbecco's modified Eagle's medium (DMEM) (Invitrogen) supplemented with $10 \%$ fetal bovine serum (FBS) (HyClone, Logan, UT, USA) and $1 \%$ penicillin/streptomycin (Invitrogen).

Plasmid and transfection. The region of the human Snail 3'UTR from 400 to $500 \mathrm{bp}$, which contained a conserved miR-153 binding site, was obtained by PCR amplification and subcloned into pEGFP-C1 (Clontech, Mountain View, CA, USA) and pGL3 vector (Promega, Madison, WI, USA). The primers selected were as follows: Snail-3'UTR-GFP-F, 5'-CCGGAATTCGATTCCTGAGCTGGCCTGTC-3' and Snail-3'UTR-GFP-R, 5'-CCGGAATTCGATTCCTGAGCTG
GCCTGTC-3'; Snail-3'UTR-luc-F, 5'-CCGCTCGAGGATTC CTGAGCTGGCCTGTC-3' and Snail-3'UTR-luc-R, 5'-ATA AGAATGCGGCCGCACACATCGGTCAGACCAGAG-3'.

The miR-153 mimics, negative control (NC) and miR-153 inhibitor were purchased from RiboBio Co., Ltd. (Guangzhou, Guangdong, China). Transfection of the plasmids, microRNA and microRNA inhibitor was performed using Lipofectamine 2000 (Invitrogen) according to the manufacturer's instructions.

Western blotting. Cells were harvested in sampling buffer (62.5 mmol/1 Tris- $\mathrm{HCl}$ (pH 6.8), 10\% glycerol, 2\% SDS) and heated for $5 \mathrm{~min}$ at $100^{\circ} \mathrm{C}$. The protein concentration was determined by the Bradford assay using a commercial kit purchased from Bio-Rad Laboratories. Equal quantities of protein were separated electrophoretically on $12 \%$ SDS/polyacrylamide gels and transferred onto polyvinylidene difluoride membranes (Roche). The membranes were probed with anti-Snail, anti-E-cadherin, anti-N-cadherin, anti-vimentin, and anti-GFP rabbit antibody (all from Cell Signaling, Danvers, MA, USA), and anti- $\alpha$-catenin mouse antibody (Abcam, Cambridge, MA, USA). Expression of target proteins was determined with horseradish peroxidase-conjugated anti-rabbit/anti-mouse $\operatorname{IgG}(1: 3,000)$ and enhanced chemiluminescence (Pierce) according to the manufacturer's suggested protocols. The membranes were stripped and reprobed with an anti- $\beta$-actin mouse monoclonal antibody (Boster BA0410) as a loading control.

RNA extraction and real-time quantitative PCR. Total miRNA from the cultured cells and fresh surgical HCC tissues was extracted using the mirVana miRNA isolation kit (Ambion, Austin, TX, USA) according to the manufacturer's instructions. cDNA was synthesized from $5 \mathrm{ng}$ of total RNA using the TaqMan miRNA reverse transcription kit (Applied Biosystems, Foster City, CA, USA), and the reaction was performed with the following parameter values: $15 \mathrm{~min}$ at $37^{\circ} \mathrm{C}, 10 \mathrm{~min}$ at $65^{\circ} \mathrm{C}, 5 \mathrm{~min}$ at $85^{\circ} \mathrm{C}$ and $-20^{\circ} \mathrm{C}$ until use. The expression levels of miR-153 were quantified using miRNA-Specific TaqMan miRNA assay kit (Applied Biosystems) on the Applied Biosystems 7500 Sequence Detection system with $20 \mu \mathrm{l}$ volume reaction containing $2 \mu \mathrm{l}$ reverse transcription product, $10 \mu \mathrm{l} 2 \mathrm{X}$ All-in-One ${ }^{\mathrm{TM}}$ qPCR mix, $2 \mu 1$ PCR forward primer $(2 \mu \mathrm{M}), 2 \mu$ l Universal

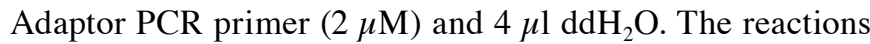
were incubated in 96 -well plates at $95^{\circ} \mathrm{C}$ for $10 \mathrm{~min}$, followed by 40 cycles, and then ramped from 66 to $95^{\circ} \mathrm{C}$ to obtain the melting curve. Each sample was analyzed in triplicate. No template and no reverse transcriptase were included as NCs. The expression of miRNA was defined based on the threshold cycle $(\mathrm{Ct})$, and relative expression levels were calculated as 2-[(Ct of miR-153) - (Ct of U6)] after normalization with reference to expression of U6 small nuclear RNA.

Migration assay. The invasion assay was conducted using a Matrigel-coated Boyden chamber of a 24-well Transwell plate consisting of 8-mm membrane filter inserts (Corning). Briefly, the cells were trypsinized and suspended in serum-free medium. Then $1.5 \times 10^{5}$ cells were added to the upper chamber coated with 50\% Matrigel (BD Biosciences), whereas the lower 


\section{A}

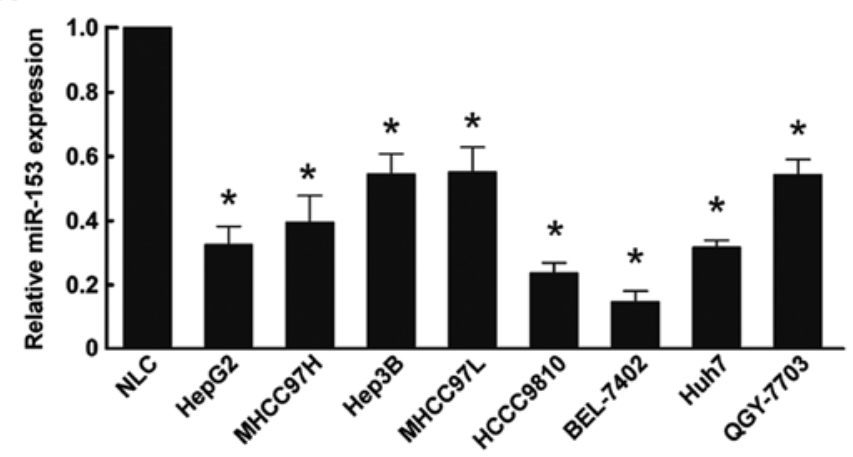

B

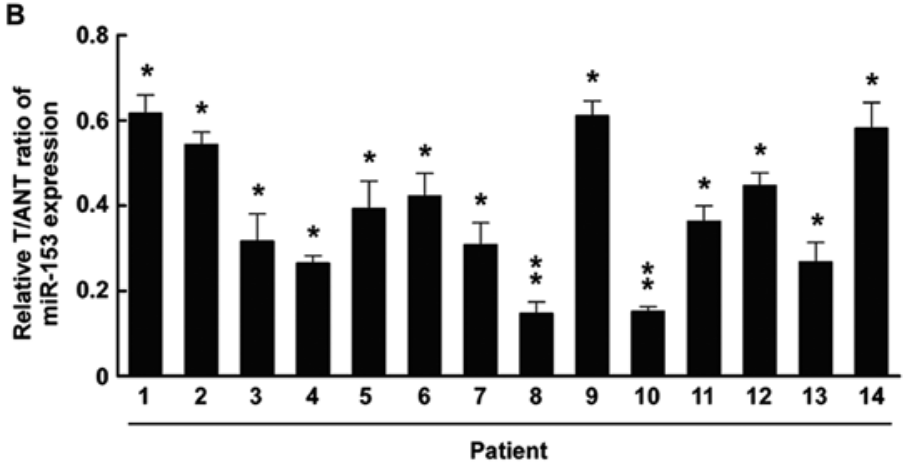

Figure 1. Downregulation of miR-153 in HCC cell lines and tissues. (A) Real-time PCR analysis of miR-153 expression in NLCs and HCC cell lines, including HepG2, MHCC97H, Hep3B, MHCC97L, HCCC9810, BEL-7402, Huh7 and QGY-7703. (B) The expression of miR-153 was examined in paired primary HCC tissues (T) with paired adjacent normal tissues (ANT) of 14 individual patients. The average miR-153 expression was normalized by U6 expression. Experiments were repeated at least 3 times. Each bar represents the mean of three independent measurements. ${ }^{*} \mathrm{P}<0.05,{ }^{* *} \mathrm{P}<0.01$. HCC, hepatocellular carcinoma; NLCs, normal liver epithelial cells.

chamber was filled with medium with $10 \%$ FBS. After incubation for $36 \mathrm{~h}$, the cells that invaded the coated membrane to the lower surface were fixed with $4 \%$ paraformaldehyde and stained with hematoxylin. Cell counting was conducted under a microscope (magnification, x20). All experiments were performed in triplicate.

Wound-healing assay. Cells were trypsinized and seeded into 6-well tissue culture plates and allowed to reach almost to confluency for $24 \mathrm{~h}$. Then the cells were serum starved $(0.5 \%$ serum) for $24 \mathrm{~h}$. Wounds were created in the confluent cells using a 100-ml pipette tip, and the cells were then cultured in complete media (10\% serum). Images of cells migrating into the wound were captured at time-points of 0,12 and $24 \mathrm{~h}$ under an inverted microscope (magnification, $\mathrm{x} 20$ ). The migration activity was calculated as the number of cells entering into the rectangle. All experiments were performed in triplicate.

$3 D$ morphogenesis assay. Dishes (24-well) were coated with growth factor reduced Matrigel (BD Biosciences) and covered with growth medium supplemented with $2 \%$ Matrigel. Cells were trypsinized and seeded at a density of $10^{4}$ cells/well, and medium was replaced with 2\% Matrigel every 3-4 days. Microscopic images were captured at 2-day intervals for 2-3 weeks.

Luciferase assays. Cells $\left(3.5 \times 10^{4}\right)$ were seeded in triplicate in 24-well plates and allowed to settle for $24 \mathrm{~h}$. One hundred nanograms of pGL3-Snail-3'UTR (wt/mut), or control-luciferase plasmid plus $1 \mathrm{ng}$ pRL-TK Renilla plasmid (Promega) were transfected into cells using Lipofectamine 2000 (Invitrogen) according to the manufacturer's recommendations. Luciferase and Renilla signals were measured $48 \mathrm{~h}$ after transfection using the Dual Luciferase Reporter assay kit (Promega) according to the manufacturer's instructions. Three independent experiments were performed and the data are presented as mean $\pm \mathrm{SD}$.

Statistical analysis. The two-tailed Student's t-test was used to evaluate the significance of the differences between two groups in all pertinent experiments. Spearman's correlation analyses were used to analyze the relationship between miR-153 expression and Snail expression levels. A P-value $<0.05$ was considered significant.

\section{Results}

miR-153 is downregulated in HCC cell lines and HCC tissues. Real-time PCR analyses revealed that miR-153 was significantly downregulated in all 8 examined HCC cell lines, including HepG2, MHCC97H, Hep3B, MHCC97L, HCCC9810, BEL-7402, Huh7 and QGY-7703, as compared with that in the NLCs (Fig. 1A). In addition, comparative analysis using real-time PCR revealed that miR-153 was differentially decreased in the 14 examined HCC biopsies paired with matched adjacent non-cancerous tissues from the same patient (Fig. 1B), indicating that miR-153 was also downregulated in HCC. 

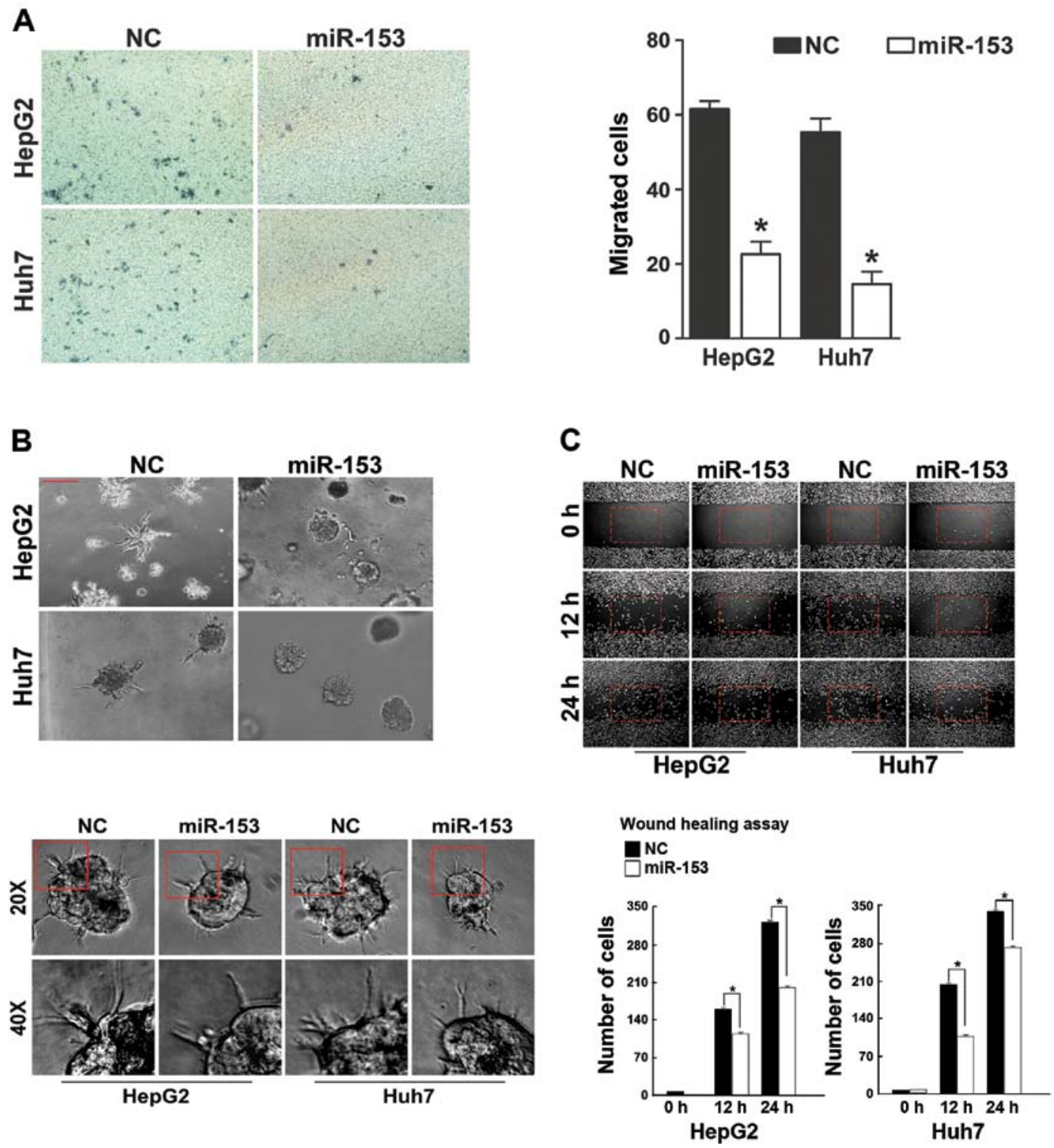

Figure 2. Upregulation of miR-153 suppresses the EMT of HCC cells. (A) The invasive properties of the HCC cells transfected with NC or miR-153 were analyzed by an invasion assay using a Matrigel-coated Boyden chamber. The migrated cells were plotted as the average number of cells per field of view from 3 different experiments, as described in Materials and methods. (B) Representative micrographs of the indicated cultured cells after an 8-day culture in the three-dimensional spheroid invasion assays. (C) The mobility of cells with NC or miR-153 was measured by testing the rate of wound closure at 0,12 and 24 h. Original magnification, x200. Error bars represent SEM (n=3). "P<0.05. EMT, epithelial-to-mesenchymal transition; HCC, hepatocellular carcinoma; NC, negative control.

Enforced expression of miR-153 suppresses migration and invasive ability of HCC cells. To investigate the biological role of miR-153 expression in the EMT phenotype and progression of HCC, we next transfected HCC cells, Huh-7 and HepG2, with hsa-miR-153 mimic oligonucleotides and examined its effect on migration and invasion. As shown in Fig. 2A, cells overexpressing hsa-miR-153 exhibited markedly decreased invasion compared with the cells transfected with $\mathrm{NC}$, as determined by Matrigel-coated Boyden chamber invasion assays. Additionally, three dimensional culture assay displayed that NC cells exhibited highly invasive behaviors, which were characterized by many filamentous-like outward pseudopodia from nearly all individual cells (Fig. 2B). In contrast, the filamentous-like pseudopodia were significantly decreased in both of the miR-153-transfected cell lines (Fig. 2B). Moreover, wound healing assays revealed that the motility and invasive- ness of the Huh-7 and HepG2 cells were markedly suppressed by overexpression of miR-153 (Fig. 2C). These results revealed that upregulation of miR-153 inhibited the EMT phenotypes of the HCC cells in vitro.

Inhibition of miR-153 promotes the migration and invasion of HCC cells. To confirm the effect of miR-153 on the EMT phenotypes of HCC cells, miR-153 expession was inhibited using the miR-153 inhibitor. As shown in Fig. 3A, Matrigel-coated Boyden chamber invasion assays demonstrated that silencing of miR-153 expression significantly increased the number of invaded HCC cells. Three dimensional culture assay showed that the miR-153 inhibitor-transfected cells exhibited a morphology typical of highly aggressive/invasive cells (Fig. 3B). Moreover, wound-healing assays demonstrated that inhibition of miR-153 markedly accelerated the motility of 
A

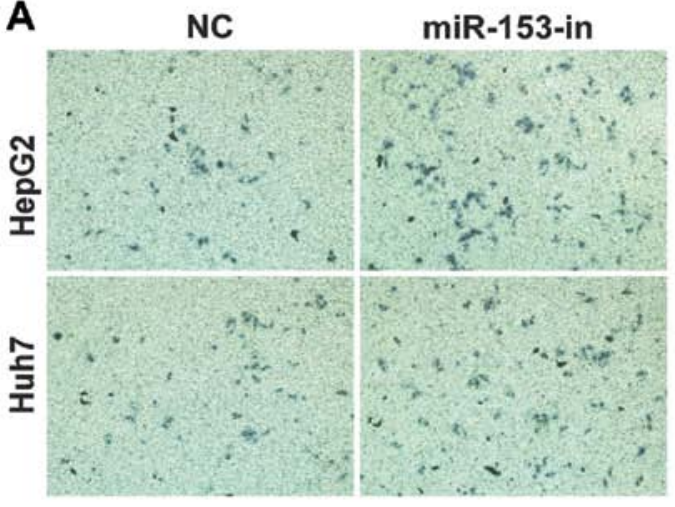

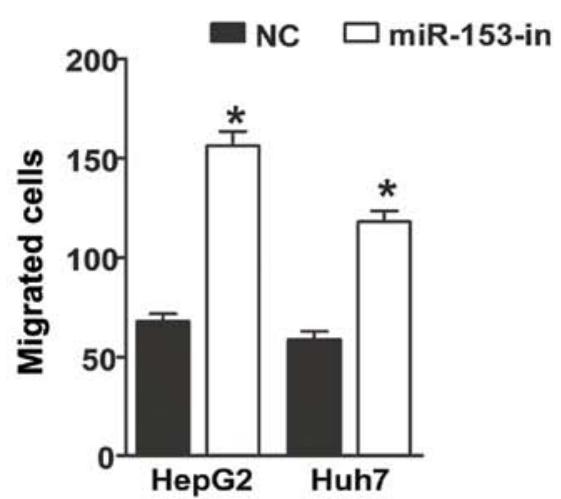

B
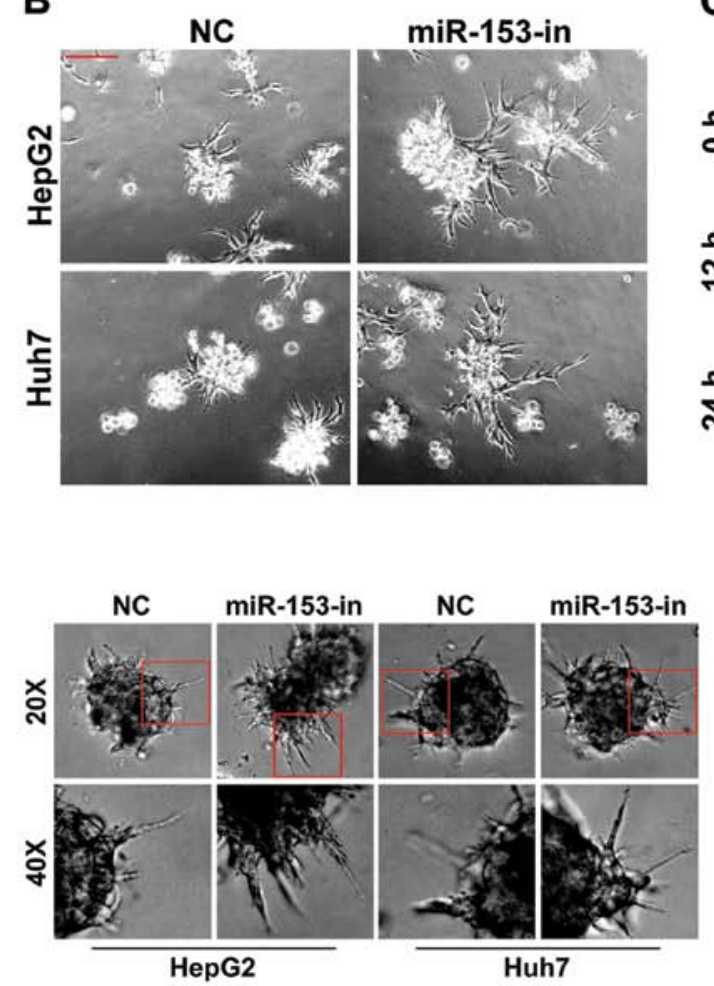

C

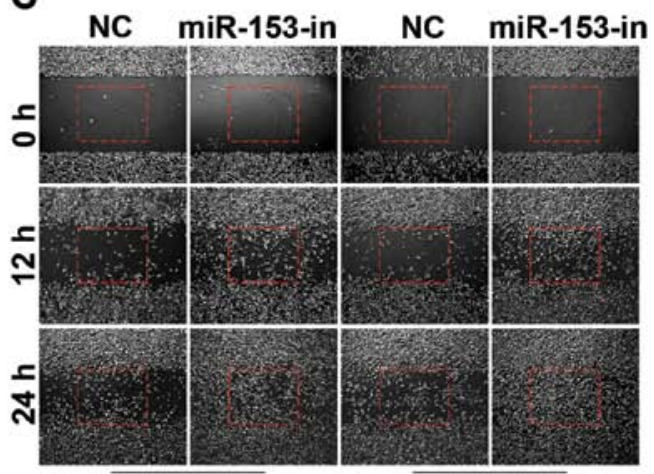

HepG2

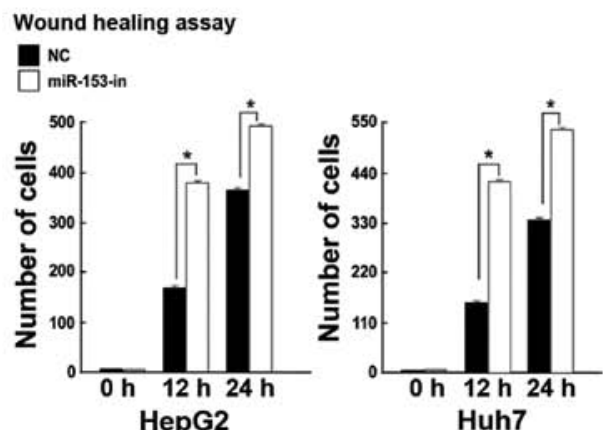

Figure 3. Inhibition of miR-153 induces the EMT of HCC cells. (A) The invasive properties of the HCC cells transfected with NC or miR-153 inhibitor were analyzed by an invasion assay using a Matrigel-coated Boyden chamber. The migrated cells were plotted as the average number of cells per field of view from 3 different measurements, as described in Materials and methods. (B) Representative micrographs of the indicated cultured cells after an 8-day culture in three-dimensional spheroid invasion assays. (C) The mobility of cells with NC or miR-153 inhibitor was measured by testing the rate of wound closure at 0,12 and $24 \mathrm{~h}$. Original magnification, $\mathrm{x} 200$. Error bars represent SEM $(\mathrm{n}=3)$. "P $<0.05$. EMT, epithelial-to-mesenchymal transition; HCC, hepatocellular carcinoma; $\mathrm{NC}$, negative control.

the HCC cells (Fig. 3C). These results indicate that inhibition of miR-153 increases the migration and invasion of HCC cells.

miR-153 directly targets the transcriptional factor Snail in HCC cells. Analysis using three publicly available microRNA target predictors (TargetScan, Pictar, and miRANDA) revealed a highly conserved region in the 3'UTR of Snail mRNA that matches the seed region of miR-153 (Fig. 4A). Western blotting confirmed that ectopic expression of miR-153 in the HepG2 and Huh7 cells decreased the expression level of Snail protein (Fig. 4B), while inhibition of miR-153 in these cells increased the Snail protein, indicating that upregulation of miR-153 results in the downregulation of Snail. We also observed that the expression of epithelial marker E-cadherin was significantly upregulated in the miR-153-overexpressing cells, while it was downregulated after miR-153 was inhibited (Fig. 4B). Conversely, ectopic expression of miR-153 evidently decreased the expression of mesenchymal markers $\mathrm{N}$-cadherin and vimentin, while inhibition of miR-153 obviously upregulated these markers (Fig. 4B). In order to examine whether miR-153 regulates Snail expression through directly targeting the predicted 3'UTR sequence, we then subcloned the 3'UTR region fragment containing the predicted miR-153 binding sites into the pEGFP-C3 and pGL3 dual luciferase reporter vectors. As shown in Fig. 4C, ectopic expression of miR-153 in the HepG2 and Huh7 cells markedly inhibited the GFP protein expression, but not the expression of GFP- $\gamma$-tubulin that was used as a control for transfection 
A

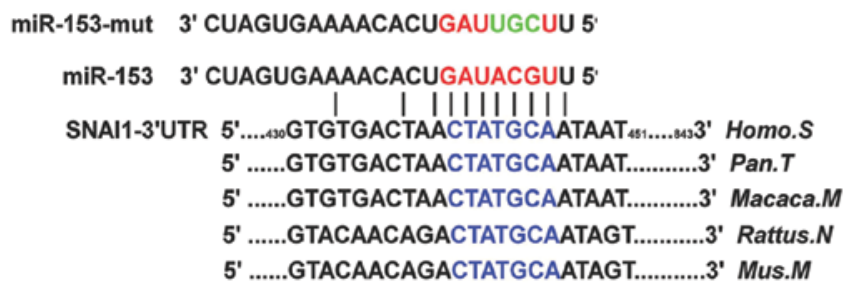

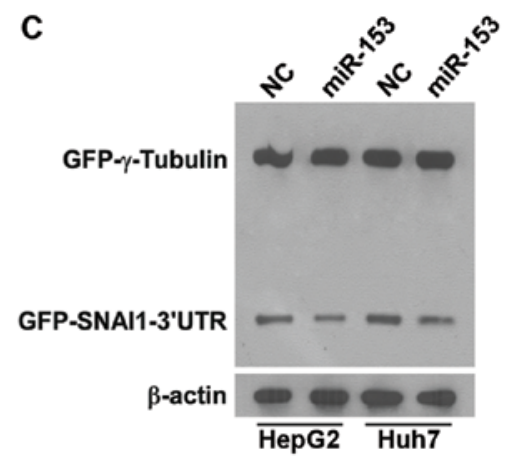

B

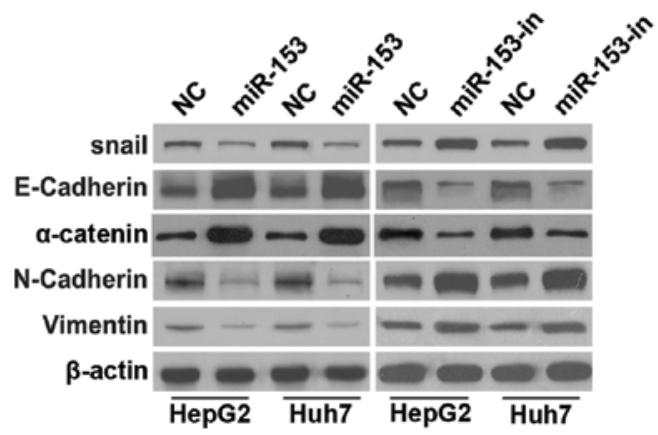

D $\quad$ NC

miR-153 mimic miR-153 inhibitor miR-153 mut

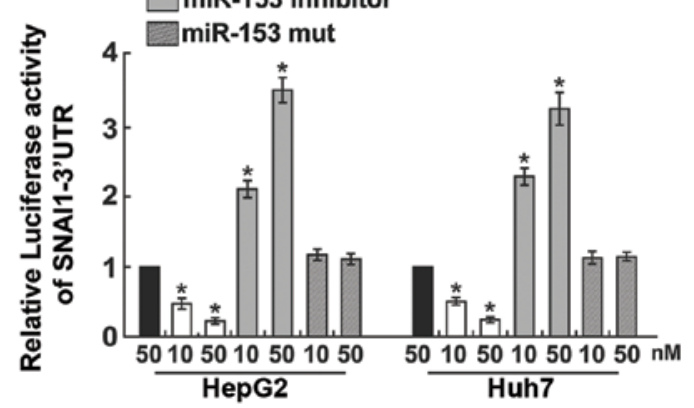

Figure 4. miR-153 downregulates Snail by directly targeting its 3'UTR. (A) Predicted miR-153 target sequences in 3'UTR of Snail (Snail) and a mutant containing four mutated nucleotides in 3'UTR of Snail. (B) Western blotting of Snail, E-cadherin, $\alpha$-catenin, N-cadherin and vimentin expression following miR-153 overexpression or miR-153 inhibition. $\beta$-actin served as the loading control. (C) Western blotting of GFP expression in the indicated cells. (D) Luciferase assay of the indicated cells transfected with the pGL3-Snail-3'UTR reporter with increasing amounts (10 and $50 \mathrm{nM}$ ) of miR-153 mimic, or miR-153 inhibitor, or miR-153 mutant. Each bar represents the mean \pm SD of three independent experiments. * $\mathrm{P}<0.05 .3^{\prime} \mathrm{UTR}, 3^{\prime}$-untranslated region.
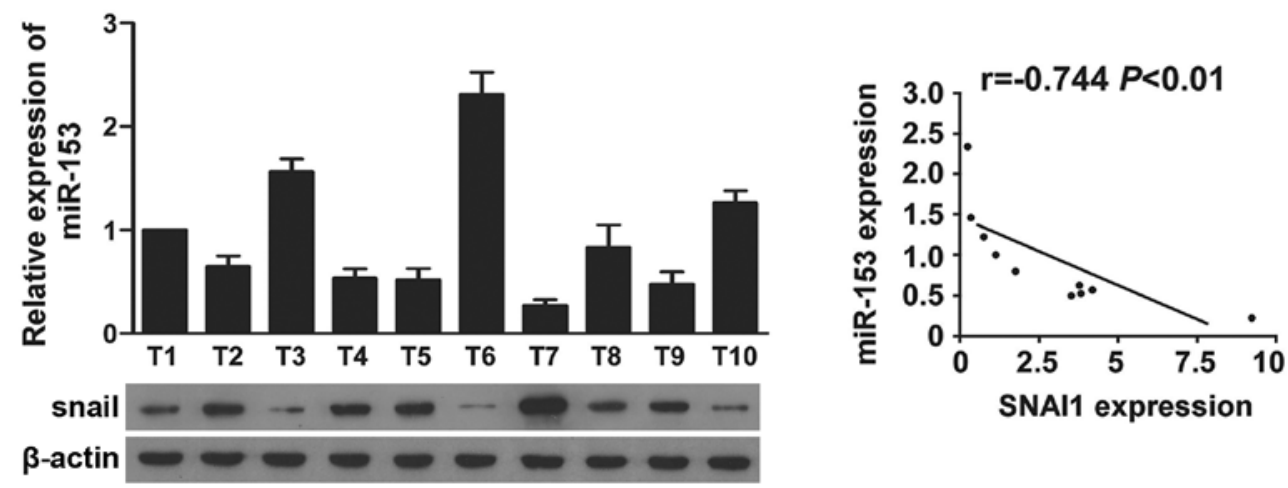

Figure 5. miR-153 expression is inversely correlated with Snail expression in the HCC tissues. Analysis of the correlation between miR-153 expression and Snail expression levels in 10 freshly collected HCC samples. Each bar represents the mean \pm SD of three independent experiments. HCC, hepatocellular carcinoma.

efficiency, suggesting that miR-153 specifically affected the Snail 3'UTR. Furthermore, a consistent and dose-dependent reduction in luciferase activity upon miR-153 transfection was observed in the HepG 2 and Huh7 cells, which was reversed by transfection with the miR-153 inhibitor (Fig. 4D). Point mutations in the tentative miR-153-binding seed region abrogated the aforementioned suppressive effect of miR-153, demonstrating that Snail is a bona fide target of miR-153 (Fig. 4D).

Clinical relevance of miR-153 and Snail expression in human $H C C$. To further examine whether the function of miR-153 in the suppression of Snail expression by targeting the 3'UTR of
Snail mRNA identified in the in vitro experiment is clinically relevant, we examined the expression levels of miR-153 and Snail in 10 cases of freshly prepared human HCC biopsies. As shown in Fig. 5A (left), the Snail protein level was relatively lower in the tissues having higher miR-153 expression. In contrast, higher levels of Snail were found in tissues with lower miR-153 expression. Furthermore, statistical analysis demonstrated that miR-153 expression was inversely correlated with Snail (Fig. 5B, r=-0.744, P<0.01), which further strengthened the notion that miR-153 inhibits the migration and invasion of HCC through suppression of the EMT modulator Snail. 


\section{Discussion}

The key finding of the present study was that miR-153 expression was noticeably downregulated in the HCC cell lines and HCC tissues as compared with that in the NLCs and matched-adjacent normal liver tissues, respectively. We further demonstrated that ectopic expression of miR-153 reduced EMT of HCC cells, while inhibition of miR-153 promoted these effects. Additionally, upregulation of miR-153 in the HCC cells led to the downregulation of epithelial markers such as E-cadherin and $\alpha$-catenin, and upregulation of mesenchymal markers such as N-cadherin and vimentin. Furthermore, we demonstrated that miR-153 decreased Snail expression by directly targeting the $3^{\prime}$ UTR of Snail mRNA. These findings suggest that deregulation of miR-153 may play a crucial role in promoting EMT and progression of human $\mathrm{HCC}$, and provide new mechanisms in Snail regulation.

HCC is the fifth most common type of cancer and the third leading cause of cancer-related mortality worldwide (26). Although both environmental factors and genetic/epigenetic alterations are considered to be major etiologies of HCC, the molecular mechanisms of its development and progression remain largely unknown (26-28). Thus, it is critical to illustrate the mechanisms underlying HCC progression. In recent years, a new class of regulatory RNAs, microRNAs, has been linked to the molecular pathogenesis of HCC $(29,30)$. Increasing evidence suggests that microRNAs can act as oncogenes or tumor suppressors (31). For instance, in HCC, miR-18 and miR-224 were significantly upregulated in HCC compared with noncancerous tissues, while miR-199a, miR-195, miR-200a and miR-125a were downregulated in HCC tissues (32). miR-101 was downregulated in HCC and its overexpression was found to promote apoptosis and suppress tumorigenicity (33). In constrast, overexpression of miR-222 promoted cell migration in HCC by activating AKT signaling (34). These observations implicate the universal role of microRNAs in carcinogenesis and malignant progression of HCC. In this study, we found that miR-153 was significantly downregulated in HCC cell lines as compared with that in the NLCs. Meanwhile, the expression of miR-153 was shown to be downregulated in HCC tissue in comparison with that in the adjacent non-cancerous tissue from the same patient. Moreover, we demonstrated that ectopic expression of miR-153 markedly suppressed the EMT phenotypes of HepG2 and Huh7 cells compared with that of the control cells, while suppression of miR-153 enhanced the cell migration and invasion of both cell lines, indicating that downregulation of miR-153 may correlate with clinical HCC progression and function as a tumor suppressor. The expression level of miR-153 was also found to be frequently upregulated in various human tumor types, including colorectal cancer, HCC and chronic myeloid leukemia cells (39-41). In disagreement with our results, it was reported that miR-153 was upregulated in prostate cancer and overexpression of miR-153 promoted cell proliferation via downregulation of the PTEN tumor-suppressor gene (35). This discrepancy in miR-153 expression in prostate cancer and HCC may reflect the distinct functions of one certain microRNA in diverse cancer types with different genetic or epigenetic backgrounds. Actually, the two faces of microRNA expression have been revealed previously. For example, miR-181b and miR-181c are upregulated in pancreas cancer but downregulated in glioblastoma (20). Recently, it was reported that downregulation of miR-153 was significantly correlated with advanced clinical stage of ovarian cancer (36). In addition, overexpression of miR-153 inhibited stretch stress-enhanced vascular smooth muscle cell proliferation and the activity of PI3K-AKT signaling (37). This observation to some degree provides evidence that supports the inhibitory role of miR-153 in the progression of human cancers. However, the majority of biological functions of miR-153 in the development and progression of human cancers have not been revealed.

In recent decades, hepatocellular EMT has been identified to contribute to liver fibrosis and play pivotal roles in the dissemination of HCC $(4,28)$. As indicated above, Snail is a powerful repressor of E-cadherin transcription and a convergent hub of EMT $(6,38)$. It has been reported that Snail expression is associated with a poor prognosis of $\mathrm{HCC}$ and its overexpression was found to accelerate HCC invasion by upregulating MMP expression $(39,40)$. Previous studies found that Snail was regulated at both the transcriptional and post-transcriptional levels (41). Signaling molecules such as TGF $\beta$, Notch and NFkB increased the transcription of Snail by directly binding to the Snail promoter $(41,42)$. Post-transcriptionally, Snail activity is mainly regulated by phosphorylation, which in turn regulates its subcellular localization $(14,41)$. GSK3 not only promoted the nuclear translocation of Snail but also led to its rapid degradation via the proteasome (43). Recently, the role of microRNAs in controlling the expression of Snail in human cancers has emerged. It was shown that miR-34, miR-30a and miR-30b inhibited EMT by targeting the 3'UTR of Snail mRNA $(44,45)$. In this study, we first observed that Snail was a theoretical target of miR-153 through bioinformatic analysis. Then, we confirmed that Snail was a bona fide target of miR-153. In addition, E-cadherin, the downstream target of Snail, was noticeably increased by the overexpression of miR-153. The luciferase activity assay and point mutation analysis confirmed that the downregulation of Snail was mediated by miR-153 through its binding to the 3'UTR of Snail mRNA.

In summary, the present study provides, for the first time, an important link between miR-153-mediated migration and invasion of HCC cells and downregulation of Snail. Elucidating the precise role of miR-153 in HCC progression and Snail modulation will not only increase our knowledge of the pathogenesis of HCC but will also assist in the establishment of novel therapeutic strategies.

\section{Acknowledgements}

This study was supported by the National Natural Science Foundation of China (grant no. 81072002).

\section{References}

1. Radisky DC: Epithelial-mesenchymal transition. J Cell Sci 118: 4325-4326, 2005.

2. Thiery JP: Epithelial-mesenchymal transitions in tumour progression. Nat Rev Cancer 2: 442-454, 2002.

3. Yang J and Weinberg RA: Epithelial-mesenchymal transition: at the crossroads of development and tumor metastasis. Dev Cell 14: 818-829, 2008.

4. van Zijl F, Zulehner G, Petz M, Schneller D, Kornauth C, Hau M, Machat G, Grubinger M, Huber H and Mikulits W: Epithelial-mesenchymal transition in hepatocellular carcinoma. Future Oncol 5: 1169-1179, 2009. 
5. Bolos V, Peinado H, Perez-Moreno MA, Fraga MF, Esteller M and Cano A: The transcription factor Slug represses E-cadherin expression and induces epithelial to mesenchymal transitions: a comparison with Snail and E47 repressors. J Cell Sci 116: 499-511, 2003

6. Cano A, Perez-Moreno MA, Rodrigo I, Locascio A, Blanco MJ, del Barrio MG, Portillo F and Nieto MA: The transcription factor snail controls epithelial-mesenchymal transitions by repressing E-cadherin expression. Nat Cell Biol 2: 76-83, 2000.

7. Comijn J, Berx G, Vermassen P, Verschueren K, van Grunsven L, Bruyneel E, Mareel M, Huylebroeck D and van Roy F: The two-handed $\mathrm{E}$ box binding zinc finger protein SIP1 downregulates E-cadherin and induces invasion. Mol Cell 7: 1267-1278, 2001.

8. Yang J, Mani SA, Donaher JL, Ramaswamy S, Itzykson RA, Come C, Savagner P, Gitelman I, Richardson A and Weinberg RA: Twist, a master regulator of morphogenesis, plays an essential role in tumor metastasis. Cell 117: 927-939, 2004.

9. Dave N, Guaita-Esteruelas S, Gutarra S, Frias A, Beltran M, Peiró $S$ and de Herreros AG: Functional cooperation between Snaill and twist in the regulation of ZEB1 expression during epithelial to mesenchymal transition. J Biol Chem 286 : 12024-12032, 2011.

10. Dhasarathy A, Phadke D, Mav D, Shah RR and Wade PA: The transcription factors Snail and Slug activate the transforming growth factor-beta signaling pathway in breast cancer. PLoS One 6: e26514, 2011.

11. De Craene B, van Roy F and Berx G: Unraveling signaling cascades for the Snail family of transcription factors. Cell Signal 17: 535-547, 2005

12. Barrallo-Gimeno A and Nieto MA: The Snail genes as inducers of cell movement and survival: implications in development and cancer. Development 132: 3151-3161, 2005.

13. Zheng B, Liang L, Wang C, Huang S, Cao X, Zha R, Liu L, Jia D, Tian Q, Wu J, et al: MicroRNA-148a suppresses tumor cell invasion and metastasis by downregulating ROCK1 in gastric cancer. Clin Cancer Res 17: 7574-7583, 2011.

14. Zhou BP, Deng J, Xia W, Xu J, Li YM, Gunduz M and Hung MC: Dual regulation of Snail by GSK-3beta-mediated phosphorylation in control of epithelial-mesenchymal transition. Nat Cell Biol 6: 931-940, 2004

15. Yang Z, Rayala S, Nguyen D, Vadlamudi RK, Chen S and Kumar R: Pak1 phosphorylation of snail, a master regulator of epithelial-to-mesenchyme transition, modulates snail's subcellular localization and functions. Cancer Res 65: 3179-3184, 2005.

16. Vinas-Castells R, Beltran M, Valls G, Gomez I, Garcia JM, Montserrat-Sentís B, Baulida J, Bonilla F, de Herreros AG and Díaz VM: The hypoxia-controlled FBXL14 ubiquitin ligase targets SNAIL1 for proteasome degradation. J Biol Chem 285: 3794-3805, 2010

17. Yamashita S, Miyagi C, Fukada T, Kagara N, Che YS and Hirano T: Zinc transporter LIVI controls epithelial-mesenchyma transition in zebrafish gastrula organizer. Nature 429: 298-302, 2004.

18. Bartel DP: MicroRNAs: genomics, biogenesis, mechanism, and function. Cell 116: 281-297, 2004

19. Ambros V: The functions of animal microRNAs. Nature 431: $350-355,2004$

20. Volinia S, Calin GA, Liu CG, Ambs S, Cimmino A, Petrocca F, Visone R, Iorio M, Roldo C, Ferracin M, et al: A microRNA expression signature of human solid tumors defines cancer gene targets. Proc Natl Acad Sci USA 103: 2257-2261, 2006.

21. Sreekumar R, Sayan BS, Mirnezami AH and Sayan AE: MicroRNA control of invasion and metastasis pathways. Front Genet 2: 58,2011.

22. Dykxhoorn DM: MicroRNAs and metastasis: little RNAs go a long way. Cancer Res 70: 6401-6406, 2010

23. Korpal M,Lee ES,Hu G and Kang Y: The miR-200 family inhibits epithelial-mesenchymal transition and cancer cell migration by direct targeting of E-cadherin transcriptional repressors ZEB and ZEB2. J Biol Chem 283: 14910-14914, 2008.

24. Gregory PA, Bert AG, Paterson EL, Barry SC, Tsykin A Farshid G, Vadas MA, Khew-Goodall Y and Goodall GJ: The miR-200 family and miR-205 regulate epithelial to mesenchymal transition by targeting ZEB1 and SIP1. Nat Cell Biol 10: 593-601, 2008.

25. Park SM, Gaur AB, Lengyel E and Peter ME: The miR-200 family determines the epithelial phenotype of cancer cells by targeting the E-cadherin repressors ZEB1 and ZEB2. Genes Dev 22: 894-907, 2008
26. El-Serag HB and Rudolph KL: Hepatocellular carcinoma: epidemiology and molecular carcinogenesis. Gastroenterology 132: 2557-2576, 2007.

27. Farazi PA and DePinho RA: Hepatocellular carcinoma pathogenesis: from genes to environment. Nat Rev Cancer 6: 674-687, 2006.

28. Severi T, van Malenstein H, Verslype $\mathrm{C}$ and van Pelt JF: Tumor initiation and progression in hepatocellular carcinoma: risk factors, classification, and therapeutic targets. Acta Pharmaco Sin 31: 1409-1420, 2010.

29. Lagos-Quintana M, Rauhut R, Lendeckel W and Tuschl T: Identification of novel genes coding for small expressed RNAs. Science 294: 853-858, 2001

30. Jopling CL, Yi M, Lancaster AM, Lemon SM and Sarnow P: Modulation of hepatitis C virus RNA abundance by a liver-specific MicroRNA. Science 309: 1577-1581, 2005.

31. Esquela-Kerscher A and Slack FJ: Oncomirs - microRNAs with a role in cancer. Nat Rev Cancer 6: 259-269, 2006.

32. Murakami Y, Yasuda T, Saigo K, Urashima T, Toyoda H, Okanoue $\mathrm{T}$ and Shimotohno K: Comprehensive analysis of microRNA expression patterns in hepatocellular carcinoma and non-tumorous tissues. Oncogene 25: 2537-2545, 2006.

33. Su H, Yang JR, Xu T, Huang J, Xu L, Yuan Y and Zhuang SM: MicroRNA-101, down-regulated in hepatocellular carcinoma, promotes apoptosis and suppresses tumorigenicity. Cancer Res 69: 1135-1142, 2009.

34. Wong QW, Ching AK, Chan AW, Choy KW, To KF, Lai PB and Wong N: MiR-222 overexpression confers cell migratory advantages in hepatocellular carcinoma through enhancing AKT signaling. Clin Cancer Res 16: 867-875, 2010.

35. $\mathrm{Wu} \mathrm{Z}, \mathrm{He} \mathrm{B}, \mathrm{He} \mathrm{J}$ and Mao X: Upregulation of miR-153 promotes cell proliferation via downregulation of the PTEN tumor suppressor gene in human prostate cancer. Prostate 73 596-604, 2013

36. Kim TH, Kim YK, Kwon Y, Heo JH, Kang H, Kim G and An HJ: Deregulation of miR-519a, 153, and 485-5p and its clinicopathological relevance in ovarian epithelial tumours. Histopathology 57: 734-743, 2010.

37. Song L, Duan P, Guo P, Li D, Li S, Xu Y and Zhou Q: Downregulation of miR-223 and miR-153 mediates mechanical stretch-stimulated proliferation of venous smooth muscle cells via activation of the insulin-like growth factor-1 receptor. Arch Biochem Biophys 528: 204-211, 2012.

38. Batlle E, Sancho E, Franci C, Dominguez D, Monfar M, Baulida J and García De Herreros A: The transcription factor snail is a repressor of E-cadherin gene expression in epithelial tumour cells. Nat Cell Biol 2: 84-89, 2000.

39. Miyoshi A, Kitajima Y, Kido S, Shimonishi T, Matsuyama S, Kitahara K and Miyazaki K: Snail accelerates cancer invasion by upregulating MMP expression and is associated with poor prognosis of hepatocellular carcinoma. Br J Cancer 92: 252-258, 2005.

40. Sugimachi K, Tanaka S, Kameyama T, Taguchi K, Aishima S, Shimada M, Sugimachi K and Tsuneyoshi M: Transcriptional repressor snail and progression of human hepatocellular carcinoma. Clin Cancer Res 9: 2657-2664, 2003.

41. Barbera MJ, Puig I, Dominguez D, et al: Regulation of Snail transcription during epithelial to mesenchymal transition of tumor cells. Oncogene 23: 7345-7354, 2004.

42. Sahlgren C, Gustafsson MV, Jin S, Poellinger L and Lendahl U: Notch signaling mediates hypoxia-induced tumor cell migration and invasion. Proc Natl Acad Sci USA 105: 6392-6397, 2008.

43. Bachelder RE, Yoon SO, Franci $C$, de Herreros AG and Mercurio AM: Glycogen synthase kinase-3 is an endogenous inhibitor of Snail transcription: implications for the epithelialmesenchymal transition. J Cell Biol 168: 29-33, 2005.

44. Kumarswamy R, Mudduluru G, Ceppi P, Muppala S, Kozlowski M, Niklinski J, Papotti M and Allgayer H: MicroRNA-30a inhibits epithelial-to-mesenchymal transition by targeting Snail and is downregulated in non-small cell lung cancer. Int J Cancer 130 2044-2053, 2012

45. Zhang J, Zhang H, Liu J, Tu X, Zang Y, Zhu J, Chen J, Dong L and Zhang J: miR-30 inhibits TGF- $\beta 1$-induced epithelial-to-mesenchymal transition in hepatocyte by targeting Snaill. Biochem Biophys Res Commun 417: 1100-1105, 2012. 